\title{
On Numerical Realization of Quasioptimal Parameter Choices in (Iterated) Tikhonov and Lavrentiev Regularization*
}

\section{T. Raus and U. Hämarik}

\author{
Institute of Mathematics, University of Tartu \\ J. Liivi 2, 50409 Tartu \\ E-mail: toomas.raus@ut.ee \\ E-mail(corresp.): uno.hamarik@ut.ee
}

Received October 3, 2008; revised November 20, 2008; published online February 25, 2009

\begin{abstract}
We consider linear ill-posed problems in Hilbert spaces with noisy right hand side and given noise level. For approximation of the solution the Tikhonov method or the iterated variant of this method may be used. In self-adjoint problems the Lavrentiev method or its iterated variant are used. For a posteriori choice of the regularization parameter often quasioptimal rules are used which require computing of additionally iterated approximations. In this paper we propose for parameter choice alternative numerical schemes, using instead of additional iterations linear combinations of approximations with different parameters.
\end{abstract}

Key words: ill-posed problem, regularization, (iterated) Tikhonov method, (iterated) Lavrentiev method, quasioptimal rules, parameter choice, numerical schemes.

\section{Introduction}

We consider the operator equation

$$
A u=f, \quad f \in R(A),
$$

where $A \in L(H, F)$ is a linear continuous operator, and $H, F$ are Hilbert spaces with corresponding inner products $(.,$.$) and norms \|$.$\| . We do not suppose that$ range $R(A)$ is closed and so in general our problem is ill-posed. The kernel $N(A)$ may be non-trivial. As usual in treatment of ill-posed problems, we suppose that instead of exact right-hand side $f \in F$ we have only an approximation $f_{\delta} \in F$ with given noise level $\delta$ : it holds $\left\|f_{\delta}-f\right\| \leq \delta$. Standard regularization method for solving problem (1.1) is the Tikhonov method

$$
u_{\alpha}=\left(\alpha I+A^{*} A\right)^{-1} A^{*} f_{\delta} .
$$

\footnotetext{
* This work was supported by the Estonian Science Foundation, Research Grant No. 7489
} 
The accuracy of this approximation may be increased by iteration. Let $m \in N$ be fixed and $u_{0}=u_{0, \alpha} \in H$ be the initial approximation. The $m$-iterated Tikhonov approximation $u_{\alpha}=u_{\alpha, m}$ is got by iterative computation

$$
u_{\alpha, i}=\left(\alpha I+A^{*} A\right)^{-1}\left(\alpha u_{\alpha, i-1}+A^{*} f_{\delta}\right), \quad(i=1,2, \ldots, m) .
$$

In case $m=1$ and $u_{0}=0$ we return to the Tikhonov approximation. If $H=F$ and $A=A^{*} \geq 0$ (the selfadjoint case) instead of Tikhonov method (1.2) and $m$-iterated Tikhonov method (1.3) usually the Lavrentiev method

$$
u_{\alpha}=(\alpha I+A)^{-1} f_{\delta}
$$

and $m$-iterated Lavrentiev method $u_{\alpha}=u_{\alpha, m}$

$$
u_{\alpha, i}=(\alpha I+A)^{-1}\left(\alpha u_{\alpha, i-1}+f_{\delta}\right), \quad(i=1,2, \ldots, m)
$$

are used. It is well known, that approximations (1.2), (1.3) have form

$$
u_{\alpha}=u_{0}+g_{\alpha}\left(A^{*} A\right) A^{*}\left(f_{\delta}-A u_{0}\right)
$$

and approximations (1.4), (1.5) have form

$$
u_{\alpha}=u_{0}+g_{\alpha}(A)\left(f_{\delta}-A u_{0}\right)
$$

with generating function $g_{\alpha}(\lambda)=(\alpha+\lambda)^{-1}$ in case of Tikhonov and Lavrentiev method and $g_{\alpha}(\lambda)=\frac{1}{\lambda}\left[1-\left(\frac{\alpha}{\alpha+\lambda}\right)^{m}\right]$ in case of iterative variants of these methods. Note that the function $g_{\alpha}(\lambda)$ satisfies the conditions (1.8)-(1.10):

$$
\begin{aligned}
& \sup _{0 \leq \lambda \leq a}\left|\sqrt{\lambda} g_{\alpha}(\lambda)\right| \leq \gamma_{*} \alpha^{-1 / 2}, \quad \alpha \geq 0, \\
& \sup _{0 \leq \lambda \leq a} \lambda^{p}\left|1-\lambda g_{\alpha}(\lambda)\right| \leq \gamma_{p} \alpha^{p}, \quad \alpha \geq 0, \quad 0 \leq p \leq p_{0}, \\
& \sup _{0 \leq \lambda \leq a}\left|g_{\alpha}(\lambda)\right| \leq \gamma \alpha^{-1}, \quad \alpha \geq 0,
\end{aligned}
$$

where $p_{0}=m$ ( $p_{0}$ is called the qualification of method), $\gamma=m, \gamma_{p}=$ $\left(\frac{p}{m}\right)^{p}\left(1-\frac{p}{m}\right)^{m-p}$ and $\gamma_{*}=1 / 2$ for Tikhonov method and $\gamma_{*}=\sqrt{m}$ in case $m \geq 2, a \geq\left\|A^{*} A\right\|$ for the approximation (1.6) and $a \geq\|A\|$ for the approximation (1.7) (see [16, 17])). For smoother solutions larger $m$ may be recommended (see Section 2: for larger $p$ in (2.3) error estimate (2.4) for larger $p_{0}=m$ may be got).

In the regularization methods of the form (1.6), (1.7) the important problem is the choice of a proper regularization parameter $\alpha$. If $\alpha$ is too small, the numerical implementation will be unstable and the approximation $u_{\alpha}$ will be useless; if $m$ is small and $\alpha$ is too big, the approximation $u_{\alpha}$ is dominated by the initial guess $u_{0}$.

This paper is devoted to several quasioptimal rules for choice of $\alpha=\alpha(\delta)$. These rules require computing of additionally iterated approximations. In this paper we propose for parameter choice alternative numerical schemes, where instead of additional iterations linear combinations of approximations with different parameters are used. The paper is organized as follows. In Sections 2, 3 the quasioptimality and corresponding rules for parameter choice are considered. Section 4 contains alternative numerical schemes for these rules. 


\section{Quasioptimality of the Parameter Choice Rule}

In the following we introduce the property of quasioptimality to characterize the quality of choice of the regularization parameter for concrete problem $A u=f$. Consider the method $\mathrm{P}$ of the form (1.6) (or form (1.7) in selfadjoint case). Let $\mathrm{R}$ be a rule for the choice of regularization parameter and let $\alpha(R)$ be the parameter chosen by Rule $\mathrm{R}$.

Definition 1 [see [13]]. Rule $\mathrm{R}$ for the a posteriori choice of the regularization parameter $\alpha=\alpha(R)$ is weakly quasioptimal (or quasioptimal), if there exists a constant $C$ (which depends not on $A, u_{*}, f_{\delta}$ ) such that for each $f_{\delta},\left\|f_{\delta}-f\right\| \leq \delta$ it holds the error estimate

$$
\begin{gathered}
\left\|u_{\alpha(R)}-u_{*}\right\| \leq C \inf _{\alpha \geq 0} \psi(\alpha)+O(\delta), \\
\psi(\alpha):= \begin{cases}\left\|\left(I-A^{*} A g_{\alpha}\left(A^{*} A\right)\right)\left(u_{0}-u_{*}\right)\right\|+\gamma_{*} \alpha^{-1 / 2} \delta, & \text { if } A^{*} \neq A, \\
\left\|\left(I-A g_{\alpha}(A)\right)\left(u_{0}-u_{*}\right)\right\|+\gamma \alpha^{-1} \delta & \text { if } A^{*}=A \geq 0 .\end{cases}
\end{gathered}
$$

Here $u_{*}$ is solution of problem (1.1), nearest to the initial approximation $u_{0}$. The constant $C$ is called the coefficient of quasioptimality.

Let us motivate Definition 1. The error of the approximation (1.6) may be presented in the form

$$
u_{\alpha}-u_{*}=\left(I-A^{*} A g_{\alpha}\left(A^{*} A\right)\right)\left(u_{0}-u_{*}\right)+g_{\alpha}\left(A^{*} A\right) A^{*}\left(f_{\delta}-f\right),
$$

from which by using $\left\|f_{\delta}-f\right\| \leq \delta$ and (1.8) we get

$$
\left\|u_{\alpha}-u_{*}\right\| \leq\left\|\left(I-A^{*} A g_{\alpha}\left(A^{*} A\right)\right)\left(u_{0}-u_{*}\right)\right\|+\gamma_{*} \alpha^{-1 / 2} \delta .
$$

Hence, the weak quasioptimality means that the error of approximate solution is less than the infimum of the upper bound (2.1), multiplied with constant $C$. A usual way to characterize the quality of the parameter choice rule for method $P_{0}$ is to prove the order-optimality of the method $P_{0}$ on the different sets of solutions. The method $P_{0}$ is order-optimal on the set $M$, if

$$
\varphi\left(\delta, M, P_{0}\right) \leq \operatorname{cinf}_{P} \varphi(\delta, M, P), \quad \varphi(\delta, M, P):=\sup _{f_{\delta} \in F, u \in M,\left\|f_{\delta}-A u\right\| \leq \delta}\left\|P f_{\delta}-u\right\| .
$$

For methods of the form (1.6) the usual way (see, for example, $[4,5,7,8,14]$ ) to prove the order-optimality of the method with a priori parameter choice $\alpha=\alpha(\delta, M)$ is to use the error estimate (2.1) and to prove that

$$
\sup _{u_{*} \in M}\left\{\left\|\left(I-A^{*} A g_{\alpha(\delta, M)}\left(A^{*} A\right)\right)\left(u_{0}-u_{*}\right)\right\|+\gamma_{*} \alpha(\delta, M)^{-1 / 2} \delta\right\} \leq \operatorname{cinf}_{P} \varphi(\delta, M, P) .
$$

These considerations about the connection between the quasioptimality of rule $R$ and the order-optimality of method $P$ with parameter choice by rule $R$ may be formulated as the following theorem.

Theorem 1 [13]. Let (2.2) holds. Let the parameter choice rule $R$ be weakly quasioptimal. Then the method $P$ with parameter choice by rule $R$ is order optimal on the set $M$. 
For example, if

$$
u_{*}-u_{0}=\left(A^{*} A\right)^{p / 2} v, \quad\|v\| \leq \varrho, \quad p>0
$$

and the rule $\mathrm{R}$ is weakly quasioptimal then by Theorem 1 it holds

$$
\left\|u_{\alpha(R)}-u_{*}\right\| \leq C \varrho^{\frac{1}{p+1}} \delta^{\frac{p}{p+1}}, \quad 0<p \leq 2 p_{0}
$$

and the regularization method with parameter choice by rule $\mathrm{R}$ is order optimal for the full range $p \in\left(0,2 p_{0}\right]$. Various other sets of solutions and corresponding order optimal error estimates may be found in $[4,5,7,8,14]$.

\section{Quasioptimal Rules for Parameter Choice}

In the following we consider the quasioptimality properties of several a posteriori parameter choice rules.

Discrepancy principle $[6,16,17]$. Let $b_{2} \geq b_{1} \geq 1$. If $\left\|A u_{0}-f_{\delta}\right\| \leq b_{2} \delta$, choose $\alpha=0$. Otherwise choose $\alpha$ for which $b_{1} \delta \leq\left\|A u_{\alpha}-f_{\delta}\right\| \leq b_{2} \delta$.

The discrepancy principle for methods (1.2)-(1.5) is not weakly quasioptimal and leads to divergence of approximations (1.4). For these methods we consider the following weakly quasioptimal modification $[1,9,10]$ of the discrepancy principle. Define function

$$
d_{M D}(\alpha):=\left\|B_{\alpha}\left(A u_{\alpha}-f_{\delta}\right)\right\|, \quad B_{\alpha}:=\left\{\begin{array}{l}
\alpha^{1 / 2}\left(\alpha I+A A^{*}\right)^{-1 / 2}, \quad \text { if } A^{*} \neq A \\
\alpha(\alpha I+A)^{-1}, \quad \text { if } A^{*}=A \geq 0 .
\end{array}\right.
$$

The modification of the discrepancy principle (MD rule). Let $b_{2} \geq$ $b_{1}>1$. If $d_{M D}(0) \leq b_{2} \delta$, choose $\alpha=0$. Otherwise choose the parameter $\alpha_{M D}=\alpha$ for which $b_{1} \delta \leq d_{M D}(\alpha) \leq b_{2} \delta$.

Note, that for Tikhonov method $(m=1)$ and its iterated approximation $u_{\alpha, m}$ it holds $d_{M D}(\alpha)=\left(A u_{\alpha, m+1}-f_{\delta}, A u_{\alpha, m}-f_{\delta}\right)^{1 / 2}$ and for Lavrentiev method and its iterative variant it holds $d_{M D}(\alpha)=\left\|A u_{\alpha, m+1}-f_{\delta}\right\|$.

The property of weak quasioptimality holds if we apply to the modified discrepancy the operators $D_{\alpha, k}$, where

$$
D_{\alpha, k}= \begin{cases}\alpha^{-k}\left(A^{*} A\right)^{k-1 / 2} A^{*} B_{\alpha}^{2 k}, & \text { if } k=1 / 2,3 / 2,5 / 2, \ldots \\ \alpha^{-k}\left(A A^{*}\right)^{k} B_{\alpha}^{2 k}, & \text { if } k=1,2, \ldots\end{cases}
$$

in general case and $D_{\alpha, k}=\alpha^{-k} A^{k} B_{\alpha}^{k}, k=1 / 2,1,3 / 2,2, \ldots$ in selfadjoint case. Denote $\varphi_{k}(\alpha)=\left\|D_{\alpha, k} B_{\alpha}\left(A u_{\alpha}-f_{\delta}\right)\right\|$ and consider the following rule.

Rule R1 $[11,12,13]$. Let $b_{1}, b_{2}$ be the constants such that $b_{2} \geq b_{1}>\widetilde{\gamma}_{k}^{h(k)}$, where $\widetilde{\gamma}_{k}^{s}=\left(\gamma_{k / \tau(s)}\right)^{\tau(s)}, \tau(s)=1+(s+1) / m$ and $h(k)=k$ in selfadjoint case, $h(k)=k-1 / 2$ in general case. Choose $\alpha(\delta)$ for which $\varphi_{k}(\alpha(\delta)) \geq b_{1} \delta$, but $\varphi_{k}(\alpha) \leq b_{2} \delta$ for each $\alpha \leq \alpha(\delta)$. 
The monotone error rule (ME rule) [15]. Choose in methods (1.2), (1.3) the parameter $\alpha_{M E}=\alpha(\delta)$ for which

$$
d_{M E}(\alpha):=\frac{\left\|B_{\alpha}\left(A u_{\alpha}-f_{\delta}\right)\right\|^{2}}{\left\|B_{\alpha}^{2}\left(A u_{\alpha}-f_{\delta}\right)\right\|}=\frac{\left(A u_{\alpha, m}-f_{\delta}, A u_{\alpha, m+1}-f_{\delta}\right)}{\left\|A u_{\alpha, m+1}-f_{\delta}\right\|}=\delta .
$$

In [15], it was proved that $\left\|u_{\alpha_{M E}}-u_{*}\right\| \leq\left\|u_{\alpha_{M D}}-u_{*}\right\|$, so the ME rule is weakly quasioptimal. The ME rule has the property $\frac{d}{d \alpha}\left\|u_{\alpha}-u_{*}\right\| \geq 0$ for all $\alpha \in\left(\alpha_{M E}, \infty\right)$, therefore $\alpha_{M E} \geq \alpha_{o p t}:=\arg \min \left\{\left\|u_{\alpha}-u_{*}\right\|, \alpha>0\right\}$. Usually $\alpha_{M E}<1$ and $\alpha_{o p t}=\alpha_{M E}^{c}$ with some constant $c \geq 1$. Our extensive numerical experiments suggest to use a not quasioptimal parameter $\alpha_{M E}^{1.1}$ instead of $\alpha_{M E}$.

In paper [3] for self-adjoint problems the analog of the ME rule was proposed, choosing in methods (1.4), (1.5) parameter $\alpha$ from equation

$$
d_{M E}(\alpha):=\frac{\left\|B_{\alpha}\left(A u_{\alpha}-f_{\delta}\right)\right\|^{2}}{\left\|B_{\alpha}^{2}\left(A u_{\alpha}-f_{\delta}\right)\right\|}=\frac{\left\|A u_{\alpha, m+1}-f_{\delta}\right\|^{2}}{\left\|A u_{\alpha, m+2}-f_{\delta}\right\|}=b \delta, \quad b>1 .
$$

In numerical experiments presented in [3], this rule gave for methods (1.4), (1.5) better results than $\mathrm{MD}$ and $\mathrm{R} 1$ rules. The following Theorems 2, 3 are proved in [13].

Theorem 2. Let $f \in R(A), H=F, A=A^{*} \geq 0,\left\|f_{\delta}-f\right\| \leq \delta$. Let the parameter $\alpha(\delta)$ in (iterated) Lavrentiev method be chosen according to the MD and $R 1$ rules. Then the error estimate

$$
\left\|u_{\alpha(\delta)}-u_{*}\right\| \leq \max \left(C_{1}\left(b_{1}\right), C_{2}\left(b_{2}\right)\right) \inf _{\alpha \geq 0} \psi(\alpha)
$$

with certain constants $C_{1}\left(b_{1}\right), C_{2}\left(b_{2}\right)$ holds.

Under some mild condition (see [3]) the last error estimate holds also, if we use in methods (1.4), (1.5) the ME rule, but in the general case the quasioptimality of the ME rule for these methods is still an open problem.

Theorem 3. Let $\left\|f_{\delta}-f\right\| \leq \delta$. Let the parameter $\alpha(\delta)$ in methods (1.2), (1.3) be chosen in case $f \in R(A)$ according to the $M D$ or $M E$ rule and in case $Q f \in R(A)$ according to the $R 1$ rule. Then the error estimate

$$
\left\|u_{\alpha(\delta)}-u_{*}\right\| \leq \max \left(C_{1}\left(b_{1}\right), C_{2}\left(b_{2}\right)\right) \inf _{\alpha \geq 0} \psi(\alpha)
$$

holds, where $b_{1}=b_{2}=1$ in case of rule (3.1) and $b_{1}=b_{2}=b$ in case of rule (3.2).

\section{Numerical Realization of Rules}

Let us assume that $q<1$. Often for numerical realization of rules MD, ME, the regularization parameter $\alpha(\delta)=\alpha_{i}$ is chosen from decreasing sequence $\alpha_{i}=m q^{i}, i=0,1,2, \ldots$, where $i$ is the smallest index with $d_{M D}\left(\alpha_{i}\right) \leq b \delta$ $(b>1), d_{M E}\left(\alpha_{i}\right) \leq b \delta(b>1)$, respectively. For realization of rule R1 from increasing sequence $\alpha_{i}=a_{i-1} / q$ regularization parameter $\alpha(\delta)$ is chosen as 
$a_{i_{0}}$, where $i_{0}+1$ is the smallest index with $\varphi_{k}\left(\alpha_{i}\right) \geq b \delta, b>\widetilde{\gamma}_{k}^{h(k)}$. We call these analogs of rules MD, ME, R1 as rules MDa, MEa and R1a. For avoiding instability of computing $\varphi_{k}(\alpha)$ for small $\alpha$ another analog of rule R1 may be used, which we call rule R1b. Here from decreasing sequence $\alpha_{i}=m q^{i}$ $(i=0,1, \ldots)$ at first $i_{0}$ as the smallest index with $d_{M D}\left(\alpha_{i}\right) \leq b \delta$ is chosen and for the regularization parameter $\alpha_{j_{0}}$ is taken, were $j_{0} \geq i_{0}$ is the smallest index with $\varphi_{k}\left(\alpha_{j_{0}}\right) \leq b \delta$. It can be shown that for these rules the error estimate

$$
\left\|u_{\alpha(\delta)}-u_{*}\right\| \leq \max \left(q^{-s} C_{1}(b), C_{2}(b)\right) \inf _{\alpha \geq 0} \psi(\alpha)+\delta
$$

holds, where $s=1 / 2$ in case of nonselfadjoint problems and $s=1$ in case of selfadjoint problems.

Considered variants of quasioptimal rules require computing of additionally iterated approximate solutions. Namely, number of required iterations in methods (1.3), (1.5) is $m+1$ for MD rule, $m+\operatorname{int}(k-1 / 2)+1$ (for (1.3)) or $m+\operatorname{int}(k)+1$ (for (1.5)) for R1 rule (where $\operatorname{int}(k)$ is integer part of $k$ ) and $m+1$ (for (1.3)) or $m+2$ (for (1.5)) for ME rule.

In the following we present formulas for approximate realization of these rules, where some linear combinations of consecutive approximate solutions $u_{\alpha_{i}, m}$ are used instead of additional iterations. For approximation of function $\varphi_{k}(\alpha)$ we note that generating function $g_{\alpha}(\lambda)$ in methods (1.3), (1.5) satisfies the equality

$$
\frac{d^{k}}{d\left(\alpha^{-1}\right)^{k}} g_{\alpha}(\lambda)=(-1)^{k+1} \frac{(m+k-1) !}{(m-1) !} \lambda^{k-1} \beta_{\alpha}^{k}(\lambda)\left(1-\lambda g_{\alpha}(\lambda)\right),
$$

where $\beta_{\alpha}(\lambda)=\alpha(\alpha+\lambda)^{-1}$. In method (1.3) due to equalities (1.6) and

$$
A u_{\alpha}-f_{\delta}=-\left(I-A A^{*} g_{\alpha}\left(A A^{*}\right)\right)\left(f_{\delta}-A u_{0}\right)
$$

we get

$$
\begin{aligned}
\frac{d^{k}}{d\left(\alpha^{-1}\right)^{k}} u_{\alpha} & =(-1)^{k+1} \frac{(m+k-1) !}{(m-1) !} A^{*}\left(A A^{*}\right)^{k-1} B_{\alpha}^{2 k}\left(A u_{\alpha}-f_{\delta}\right) \\
& =(-1)^{k+1} \frac{(m+k-1) !}{(m-1) !} \alpha^{k-1 / 2} D_{\alpha, k-1 / 2} B_{\alpha}\left(A u_{\alpha}-f_{\delta}\right),
\end{aligned}
$$

therefore

$$
\varphi_{k}(\alpha)=\left\{\begin{array}{l}
C_{m, k-1 / 2} \alpha^{-k}\left\|\frac{d^{k+1 / 2} u_{\alpha}}{d\left(\alpha^{-1}\right)^{k+1 / 2}}\right\|, \quad \text { if } k=\frac{1}{2}, \frac{3}{2}, \frac{5}{2}, \ldots, \\
\frac{C_{m, k-1}}{(m+k)^{1 / 2} \alpha^{k}}\left(\frac{d^{k} u_{\alpha}}{d\left(\alpha^{-1}\right)^{k}}, \frac{d^{k+1} u_{\alpha}}{d\left(\alpha^{-1}\right)^{k+1}}\right)^{1 / 2}, \text { if } k=1,2, \ldots,
\end{array}\right.
$$

where $C_{m, k}=(m-1) ! /(m+k) !$. Analogously we get for method (1.5)

$$
\begin{aligned}
\frac{d^{k}}{d\left(\alpha^{-1}\right)^{k}} u_{\alpha} & =(-1)^{k+1} \frac{(m+k-1) !}{(m-1) !} A^{k-1} B_{\alpha}^{k}\left(A u_{\alpha}-f_{\delta}\right) \\
& =(-1)^{k+1} \frac{(m+k-1) !}{(m-1) !} \alpha^{k-1} D_{\alpha, k-1} B_{\alpha}\left(A u_{\alpha}-f_{\delta}\right),
\end{aligned}
$$


therefore

$$
\varphi_{k}(\alpha)=\left\{\begin{array}{c}
C_{m, k} \alpha^{-k}\left\|\frac{d^{k+1} u_{\alpha}}{d\left(\alpha^{-1}\right)^{k+1}}\right\|, \quad \text { if } k=0,1,2, \ldots \\
\frac{C_{m, k-1 / 2}}{(m+k-1 / 2)^{1 / 2} \alpha^{k}}\left(\frac{d^{k+1 / 2} u_{\alpha}}{d\left(\alpha^{-1}\right)^{k+1 / 2}}, \frac{d^{k+3 / 2} u_{\alpha}}{d\left(\alpha^{-1}\right)^{k+3 / 2}}\right)^{1 / 2}, \\
\text { if } k=\frac{1}{2}, \frac{3}{2}, \frac{5}{2}, \ldots .
\end{array}\right.
$$

For approximate computing of $\frac{d^{k} u_{\alpha}}{d\left(\alpha^{-1}\right)^{k}}$ some formula of numerical differentiation may be used. For example, on the bases of the Lagrange interpolation polynom we have

$$
f^{(k)}(x) \approx P^{(k)}(x), \quad P(x)=\sum_{i=0}^{n} \frac{w(x) f\left(x_{i}\right)}{\left(x-x_{i}\right) w^{\prime}\left(x_{i}\right)}, \quad w(x)=\prod_{i=0}^{n}\left(x-x_{i}\right) .
$$

Taking $n=k$ and using knots $\alpha_{i}=q^{i-j} \alpha_{j}, i \in\{j-s(k), \ldots, j-s(k)+k\}$, where $s(k)=k / 2$ in case of even $k$ and $s(k)=(k+1) / 2$ in case of odd $k$, we have

$$
\begin{aligned}
& \left.\frac{d^{k} u_{\alpha}}{d\left(\alpha^{-1}\right)^{k}}\right|_{\alpha=\alpha_{j}} \approx k ! \sum_{i=-s(k)}^{-s(k)+k} \frac{1}{w^{\prime}\left(\alpha_{j+1}^{-1}\right)} u_{\alpha_{j+i}}=k ! \alpha_{j}^{k} \sum_{i=-s(k)}^{-s(k)+k} \frac{1}{w_{i, k}(q)} u_{\alpha_{j+i}}, \\
& w_{i, k}=w_{i, k}(q)=\prod_{t=-s(k), t \neq i}^{-s(k)+k}\left(q^{-i}-q^{-t}\right) .
\end{aligned}
$$

It allows us to use in rule R1 for methods $(1.2),(1.3) \widetilde{\varphi}_{k}\left(\alpha_{j}\right)$ instead of $\varphi_{k}\left(\alpha_{j}\right)$, where

$$
\widetilde{\varphi}_{k}\left(\alpha_{j}\right)=\left\{\begin{array}{l}
C_{m, k-1 / 2}(k+1 / 2) ! \sqrt{\alpha_{j}} s_{k, j}, \\
s_{k, j}:=\left\|\sum_{i=-s(k+1 / 2)}^{-s(k+1 / 2)+k+1 / 2} \frac{1}{w_{i, k+1 / 2}} u_{\alpha_{j+i}}\right\|, \text { if } k=\frac{1}{2}, \frac{3}{2}, \frac{5}{2}, \ldots \\
C_{m, k-1}(m+k)^{-1 / 2} k !(k+1)^{1 / 2} \sqrt{\alpha_{j}} s_{k, j}, \\
s_{k, j}:=\left(\sum_{i=-s(k)}^{-s(k)+k} \frac{1}{w_{i, k}} u_{\alpha_{j+i}}, \sum_{i=-s(k+1)}^{-s(k+1)+k+1} \frac{1}{w_{i, k+1}} u_{\alpha_{j+i}}\right)^{1 / 2}, \\
\text { if } k=0,1,2, \ldots
\end{array}\right.
$$

In methods $(1.4),(1.5)$, instead of $\varphi_{k}\left(\alpha_{j}\right)$ we use

$$
\widetilde{\varphi}_{k}\left(\alpha_{j}\right)=\left\{\begin{array}{l}
C_{m, k-1}(k+1) ! \alpha_{j}\left\|\sum_{i=-s(k+1)}^{-s(k+1)+k+1} \frac{1}{w_{i, k+1}} u_{\alpha_{j+i}}\right\|, \quad \text { if } k=0,1, \ldots \\
C_{m, k-1}(m+k+1 / 2)^{-1 / 2}(k+1 / 2) !(k+3 / 2)^{1 / 2} \alpha_{j} s_{k j}, \\
s_{k j}:=\left(\sum_{i=-s\left(k+\frac{1}{2}\right)}^{-s\left(k+\frac{1}{2}\right)+k+\frac{1}{2}} \frac{1}{w} u_{\alpha_{j+i}}, \sum_{i=-s+\frac{1}{2}}^{-s\left(k+\frac{3}{2}\right)+k+\frac{3}{2}} \frac{1}{w_{i, k+\frac{3}{2}}} u_{\alpha_{j+i}}\right)^{1 / 2}, \\
\text { if } k=\frac{1}{2}, \frac{3}{2}, \frac{5}{2}, \ldots
\end{array}\right.
$$


For numerical realization of MD rule and ME rule in methods (1.2)-(1.5) we use extrapolation. The extrapolated approximation is a linear combination of original approximations $u_{\alpha_{i}, j}$ with $n$ different parameters $\alpha_{i}$ and has qualification $p_{0}=m n$, if $j=1, \ldots, m$ are used, and $p_{0}=m+n-1$, if only $j=m$ is used (see [2]). Therefore for source-like solutions (2.3) with $p>2 m$ the accuracy (2.4) of extrapolated approximations is much higher than for approximations (1.2)-(1.5). However, in this paper we use extrapolation not for construction of alternative approximate solution, but only for choice of $\alpha$ in methods (1.2)-(1.5). Namely, instead of computing $u_{\alpha, m+1}$ it is sufficient to use another approximation

$$
v_{\alpha}=\left(1-q^{-m}\right)^{-1} u_{\alpha / q, m}+\left(1-q^{m}\right)^{-1} u_{\alpha, m}
$$

with the same qualification $m+1$.

Proposition 1. Let $q<1$. Then the following inequalities are true:

$$
\begin{aligned}
& \left\|A u_{\alpha, m+1}-f_{\delta}\right\| \leq\left\|A v_{\alpha}-f_{\delta}\right\| \leq\left\|A u_{\alpha / q, m+1}-f_{\delta}\right\| \\
& \begin{aligned}
\left(A u_{\alpha, m+1}-f_{\delta}, A u_{\alpha}-f_{\delta}\right) & \leq\left(A v_{\alpha}-f_{\delta}, A u_{\alpha}-f_{\delta}\right) \\
& \leq\left(A u_{\alpha / q, m+1}-f_{\delta}, A u_{\alpha}-f_{\delta}\right)
\end{aligned}
\end{aligned}
$$

Proof. Let $Q(\lambda)$ be the spectral family of projectors of operator $A A^{*}$. Then (4.1) gives

$$
\left\|A u_{\alpha, m}-f_{\delta}\right\|^{2}=\int_{0}^{\left\|A A^{*}\right\|}\left(1-\lambda g_{\alpha}(\lambda)\right)^{2} d\left\|Q(\lambda)\left(A u_{0}-f_{\delta}\right)\right\|^{2} .
$$

Taking into account the equality $1-\lambda g_{\alpha / q}(\lambda)=(1+\mu)^{-m}$ with $\mu=\lambda /(\alpha q)$, for proving (4.2) it is sufficient to show that for all $\mu>0$

$(1+\mu / q)^{-m-1} \leq\left(1-q^{-m}\right)^{-1}(1+\mu)^{-m}+\left(1-q^{m}\right)^{-1}(1+\mu / q)^{-m} \leq(1+\mu)^{-m-1}$

i.e. $\left(\frac{1+\mu}{q+\mu}\right)^{m+1} q^{m+1} \leq \frac{1+\mu}{q^{-m}-1}\left[\left(\frac{1+\mu}{q+\mu}\right)^{m}-1\right] \leq 1$. Denoting $x:=(1+\mu) /(q+$ $\mu$ ) (then $\left.1 \leq x \leq q^{-1}\right)$ these inequalities take form $(x q)^{m+1} \leq(1-q)\left(q^{-m}-\right.$ $1)^{-1}\left(x^{m}+x^{m-1}+\ldots+x\right) \leq 1$. It is easy to check that these inequalities hold. Thus inequality (4.2) is proved. The proof for inequality (4.3) is similar.

For numerical realization of MD and ME rules in methods (1.2), (1.3) one can use approximations

$$
\begin{aligned}
& d_{M D}(\alpha):=\left\|B_{\alpha}\left(A u_{\alpha}-f_{\delta}\right)\right\| \approx\left(A u_{\alpha}-f_{\delta}, A v_{\alpha}-f_{\delta}\right)^{1 / 2}=: \widetilde{d}_{M D}(\alpha), \\
& \widetilde{d}_{M E}(\alpha):=\left(A u_{\alpha}-f_{\delta}, A v_{\alpha}-f_{\delta}\right) /\left\|A v_{\alpha}-f_{\delta}\right\|,
\end{aligned}
$$

respectively. Next, we take into account that in methods (1.4), (1.5)

$$
d_{M D}(\alpha)=\varphi_{0}(\alpha)=\frac{1}{m}\left\|\frac{d u_{\alpha}}{d\left(\alpha^{-1}\right)}\right\|
$$


and in method (1.4) $\left\|B_{\alpha}^{2}\left(A u_{\alpha}-f_{\delta}\right)\right\|$ can be approximated by

$$
\left\|B_{q \alpha} B_{\alpha / q}\left(A u_{\alpha}-f_{\delta}\right)\right\|=\frac{1}{(1 / q-1)\left(1-q^{2}\right)}\left\|q u_{\alpha / q}-(1+q) u_{\alpha}+u_{q \alpha}\right\| .
$$

Then for MD and for ME rules instead of functions $d_{M D}(\alpha)$ and $d_{M E}(\alpha)$ their approximations

$$
\widetilde{d}_{M D}(\alpha):=\widetilde{\varphi}_{0}(\alpha)=\frac{\alpha\left\|u_{\alpha}-u_{\alpha / q}\right\|}{m \cdot(1-q)}, \quad \widetilde{d}_{M E}(\alpha):=\frac{\alpha(1+q)\left\|u_{\alpha}-u_{\alpha / q}\right\|^{2}}{q\left\|q u_{\alpha / q}-(1+q) u_{\alpha}+u_{q \alpha}\right\|}
$$

may be used. For proving the quasioptimality of rules MDa, MEa and R1a, the following result is useful.

Theorem 4. Let for parameter choice rules $M D, M E$ or R1 from condition $b_{1} \delta \leq d(\alpha) \leq b_{2} \delta$ the error estimate

$$
\left\|u_{\alpha(\delta)}-u_{*}\right\| \leq \max \left(C_{1}\left(b_{1}\right), C_{2}\left(b_{2}\right)\right) \inf _{\alpha \geq 0} \psi(\alpha)
$$

hold. Let $\alpha_{j}=q \alpha_{j-1}, q<1, j=1,2, \ldots$ and the inequalities

$$
c_{0} d\left(\alpha_{s+1}\right) \leq \widetilde{d}\left(\alpha_{s}\right) \leq d\left(\alpha_{s+j}\right), \quad i \geq j
$$

hold. If for regularization parameter $\alpha(\delta)=\alpha_{s+i}$ is taken, where $s$ is the smallest index with inequality $\widetilde{d}\left(\alpha_{s}\right) \leq b \delta, b \geq b_{1}$, then the error estimate

$$
\left\|u_{\alpha(\delta)}-u_{*}\right\| \leq \max \left(q^{-(i-j+1) t} C_{1}(b), C_{2}\left(b c_{0}^{-1}\right)\right) \inf _{\alpha \geq 0} \psi(\alpha)+q^{-i t} \gamma \alpha_{0}^{-t} \delta
$$

holds with $t=1$ for methods (1.4), (1.5) and with $t=1 / 2$ for methods (1.2), (1.3).

The proof of this theorem is analogous to the proof of Theorem 5 in [13].

Using inequalities (4.3) one can show, that in methods (1.2)-(1.5) the inequalities $d_{M D}\left(\alpha_{s}\right) \leq \widetilde{d}_{M D}\left(\alpha_{s}\right) \leq d_{M D}\left(\alpha_{s+1}\right)$ hold. Therefore according to Theorem 3 the MDa rule with function $\widetilde{d}_{M D}(\alpha)$ is quasioptimal. Note also that in methods $(1.2),(1.4)$ the function $\widetilde{d}_{M D}(\alpha)$ has the form $\widetilde{d}_{M D}(\alpha)=$ $\| B_{\alpha / q}\left(A u_{\alpha}-f_{\delta} \|\right.$.

Note that rule R1a with function $\widetilde{\varphi}_{1 / 2}(\alpha)$ in methods (1.2), (1.3) and rule MDa with function $\widetilde{d}_{M D}(\alpha)$ in methods (1.4), (1.5) are close to the balancing principle (see $[2,5,8,13]$ ), where for regularization parameter $\alpha_{k}=q^{-k} \alpha_{0}$ $(q<1)$ is taken with smallest $k$, for which the condition

$$
m^{-1}(1-q)^{-1} \alpha_{k}^{s}\left\|u_{\alpha_{k}}-u_{\alpha_{k+1}}\right\| \geq \text { const } \delta
$$

holds. Here $s=1 / 2$ for methods (1.2), (1.3) and $s=1$ for methods (1.4), (1.5). The only difference is that in rules $\mathrm{R} 1 \mathrm{a}$ and MDa for regularization parameter $\alpha_{k-1}$ is taken. In paper [13], it is shown that by choice $\alpha(\delta)=\alpha_{k-1}$ rules R1a $(k=1 / 2)$ and MDa are quasioptimal. 


\section{References}

[1] H. Gfrerer. An a posteriori parameter choice for ordinary and iterated Tikhonov regularization of ill-posed problems leading to optimal convergence rates. Math. Comp., 49(180):507-522, 1987.

[2] U. Hämarik, R. Palm and T. Raus. Use of extrapolation in regularization methods. Journal of Inverse and Ill-Posed Problems, 15(3):277-294, 2007.

[3] U. Hämarik, T. Raus and R. Palm. On the analog of the monotone error rule for parameter choice in the (iterated) Lavrentiev regularization. Computational Methods in Applied Mathematics, 8(3):237-252, 2008.

[4] T. Hohage. Regularization of exponentially ill-posed problems. Numerical Functional Analysis and Optimization, 21(3):439-464, 2000.

[5] P. Mathé and S. V. Pereverzev. Geometry of linear ill-posed problems in variable Hilbert scales. Inverse Problems, 19(3):789-803, 2003.

[6] V. A. Morozov. On the solution of functional equations by the method of regularization. Soviet Math. Dokl., 7:414-417, 1966.

[7] M. T. Nair, S. V. Pereverzev and U. Tautenhahn. Regularization in Hilbert scales under general smoothing conditions. Inverse Problems, 21(6):1851-1869, 2005.

[8] S. V. Pereverzev and E. Schock. On the adaptive selection of the parameter in the regularization of ill-posed problems. SIAM J. Numerical Analysis, 43(5):20602076, 2005.

[9] T. Raus. Residue principle for ill-posed problems. Acta et Comment. Univ. Tartuensis, 672:16-26, 1984. (in Russian)

[10] T. Raus. On the discrepancy principle for solution of ill-posed problems with nonselfadjoint operators. Acta et Comment. Univ. Tartuensis, 715:12-20, 1985. (in Russian)

[11] T. Raus. An a posteriori choice of the regularization parameter in case of approximately given error bound of data. Acta et Comment. Univ. Tartuensis, 913:73-87, 1990

[12] T. Raus. About regularization parameter choice in case of approximately given error bounds of data. Acta et Comment. Univ. Tartuensis, 937:77-89, 1992.

[13] T. Raus and U. Hämarik. On the quasioptimal regularization parameter choices for solving ill-posed problems. Journal of Inverse and Ill-Posed Problems, 15(4):419-439, 2007.

[14] U. Tautenhahn. Optimality for ill-posed problems under general source conditions. Numer. Funct. Anal. Optim., 19(3-4):377-398, 1998.

[15] U. Tautenhahn and U. Hämarik. The use of monotonicity for choosing the regularization parameter in ill-posed problems. Inverse Problems, 15(6):14871505, 1999.

[16] G. Vainikko and A. Veretennikov. Iteration Procedures in Ill-Posed Problems. Nauka, Moscow, 1986. (in Russian)

[17] G. M. Vainikko. The principle of the residual for a class of regularization methods. USSR Comput. Math. Math. Phys., 22(3):1-19, 1982. 